ESAIM: PROCEEDINGS, October 2007, Vol. 22, 156-161

Gabriel Caloz \& Monique Dauge, Editors

\title{
MINISYMPOSIUM OPTIMISATION DE FORME: AVANT-PROPOS
}

\author{
Alain DervieuX ${ }^{1}$ AND JAN SOKOLOWSKI ${ }^{2}$
}

\begin{abstract}
Starting from Hadamard's and Garabedian's works, the shape optimisation was a part of classical calculus of variations. The analysis of free boundary problem problems is performed as well in the framework of the shape optimization. The most advanced applications are now in computer aided design of optimal shapes in many domains including car and aircraft industries. In the 80's, J.-L. Lions suggested to use the adjoint state equations for aerodynamics shape optimization problems, which leads to efficient numerical methods. Optimal Control for shapes is now popular domain in Applied Mathematics and Engineering. New shape gradients were proposed for numerical solutions of such problems (deformation velocity of J. Céa and J.-P. Zolésio; topological derivatives of shape functionals of J. Sokolowski and A. Zochowski, M. Masmoudi based on the asymptotic analysis of the Russian school) and the adjoint states are presently routinely used for numerical methods of shape optimization. Today, parameterization is an important issue for general representation and for computational efficiency. Topological derivatives were recently combined with Level Set methods. The foreword of this minisymposium review shortly these advances and try to identify some unsolved directions. Then the first communication presents this approach for a variational inequality. The aerodynamics-oriented methods are studied in three subsequent communications. First, a Free-Form-Deformation is combined with an adaptative Bézier representation of the boundary. Second, a multilevel optimisation relying on Bézier representation is studied. Then the impact of flow and functional smoothness for supersonic flows is analysed.
\end{abstract}

\footnotetext{
${ }^{1}$ INRIA Tropics Project-Team, 2004 route des lucioles, BP 93, 06902 Sophia-Antipolis, FRANCE

${ }^{2}$ Institut Elie Cartan, Laboratoire de Mathématiques, Université Henri Poincaré Nancy I, B.P. 239, 54506 Vandoeuvre-lès-Nancy cedex, FRANCE

(C) EDP Sciences, SMAI 2007
} 
Résumé. L'optimisation de forme a vraiment dépassé le stade du calcul variationnel (Hadamard) et de l'analyse des problèmes à frontière libre (Garabedian) lorsque l'on s'est avisé que le gradient et l'ordinateur sont les moyens modernes d'atteindre effectivement des formes optimales. Dans les années 80, Jacques-Louis Lions s'étonnait que l'on utilise si peu l'état adjoint en aérodynamique. De fait, le formalisme du contrôle optimal est désormais adopté pour l'optimisation de forme. Le gradient de forme a donné lieu à de nouvelles formulations comme les champs de vitesse de déformation (J. Céa, J.-P. Zolésio) puis les dérivées topologiques. En même temps, l'état adjoint a trouvé une place centrale en optimisation aérodynamique. Les méthodes de paramétrage reviennent en scène avec deux questions importantes, la généralité du paramétrage et son efficacité. Les dérivations topologiques associées depuis peu avec un paramétrage Level Set permettent de rechercher des formes dont on ignore a priori la topologie (nombre de composantes, nombre de trous,...). L'avant-propos de ce minisymposium passe rapidement en revue ces avancées et essaie d'identifier quelques questions nonrésolues. La première communication de ce minisymposium présente la dérivation topologique d'un système régi par une inéquation variationnelle et son adaptation à l'optimisation de forme Level Set. Les trois communications suivantes sont plus concernées par les questions d'efficacité car elles traitent de l'optimisation de formes aérodynamiques. La seconde communication propose un paramétrage par déformation (Free-Form-Deformation) combiné avec une représentation de la frontière par courbes de Bézier adaptative. La troisième communication explore les potentialités d'une approche multiniveau reposant sur le paramétrage Bézier. La dernière communication étudie la relation entre régularité et utilisation du gradient de forme pour des écoulements supersoniques.

\section{INTRODUCTION}

Si le dispositif ou le mécanisme est à la base de l'invention, l'ingénieur a toujours été fasciné par les questions de forme de l'objet conçu. Il est sans doute sensible à la rencontre à cette occasion entre art plastique et mathématiques. Associer forme et mathématiques, c'est notamment poser le problème de la représentation mathématique de la forme, son paramétrage. C'est ensuite maitriser la dépendance du système considéré par rapport au paramètre de forme. C'est le problème de la sensitivité pour parler un peu anglo-saxon. L'évaluation de cette sensitivité donne finalement accès à des algorithmes efficaces de contrôle par la forme. Nous passons en revue successivement ces différents aspects et quelques unes des avancées réalisées depuis les temps légendaires du début de cette discipline.

\section{Paramétrage De la forme}

\subsection{Paramétrage fonctionnel}

Si on a pensé très tôt à représenter des formes à l'aide de graphes de fonctions, le Calcul des Variations a incité à les représenter comme des perturbations d'une forme initiale soit par un déplacement de la courbe/surface normalement à elle-même (Hadamard [10]) soit par une déformation de l'espace (méthodes des variations intérieures de Garabedian [8], travaux de Murat et Simon [15]). La principale motivation de Garabedian dans son étude était la résolution de problèmes de jets à frontière libre.

Avec le progrès des premiers ordinateurs, on a su simuler de plus en plus de processus physiques dans des géométries réalistes, et on a voulu optimiser la conception d'un objet ou identifier la géométrie d'un phénomène physique inaccessible (une nappe souterraine de pétrole, par exemple). Les recherches sur la variation des domaines a redémarré avec pour problématique le contrôle optimal.

La déformation de l'espace a pris une dimension dynamique avec les dérivées matérielles de Céa et Zolesio [28]. Toutes ces représentations n'envisagent pas de changement de topologie. Ce changement est au contraire pris en compte avec le développement des méthodes topologiques [26] et les représentations par courbes de niveau [1]. 


\subsection{Paramétrage discret, paramétrage multiniveau}

Si on conçoit bien la discrétisation des paramétrages précédents sur des maillages, ce n'est pas la seule option intéressante. D'autres paramétrisations nous sont soufflées par les innovations issues de l'industries et notamment les fonctions de Casteljau-Bézier jouent un rôle non-négligeable en vertu de leur usage en CAO. Voir par exemple [6]. Ces discrétisations peuvent exiger de très nombreux degrés de liberté et produire des systèmes d'optimalité de grande raideur. Le traitement de ce point difficile peut reposer sur des préconditionnements ou des procédures multiniveau [12].

\section{Continuité}

Une fois la géométrie décrite, on suppose que l'on sait en déduire l'état du système et la fonctionnelle coût. L'analyse théorique du problème de contrôle peut être réalisée à partir des propriétés de continuité de la fonctionnelle. Les problèmes théoriques d'existence d'un contrôle optimal tiennent une importante place et font le tour des propriétés de continuité vis à vis de différentes topologies, combinées avec des propriété de compacité (ou de manière équivalente de régularité à la Tychonov de la fonctionnelle). Mentionnons spécialement les ensembles de domaines compacts par régularité de leur frontière (propriété de cône, [4]) et des travaux plus récents $[3,7]$ dans lesquels les contraintes géométriques s'expriment en nombres entiers .

Les problèmes de continuités se heurtent aux frontières de ce que l'on connait sur les équations d'état ellesmêmes. Nous l'illustrons sur une avancée récente en forme optimale pour l'aérodynamique.

On suppose que le gaz visqueux occupe le domaine doublement connexe $\Omega=B \backslash S$, où $B \subset \mathbb{R}^{3}$ est l'univers contenant tous les domaines, dont la frontière $\Sigma=\partial B$ est régulière et $S \subset B$ est un obstacle compact. De plus, on suppose que la vitesse du gaz cö̈ncide avec un champ de vecteurs donné $\mathbf{U} \in C^{\infty}\left(\mathbb{R}^{3}\right)^{3}$ sur la surface $\Sigma$. Dans ce cadre, la frontière du domaine d'écoulement $\Omega$ est divisée en trois sous-ensembles, l'admission $\Sigma_{\text {in }}$, le domaine sortant $\Sigma_{\text {out }}$, et l'ensemble caractéristique $\Sigma_{0}$, qui sont définis par les égalités

$$
\Sigma_{\text {in }}=\{x \in \Sigma: \mathbf{U} \cdot \mathbf{n}<0\}, \quad \Sigma_{\text {out }}=\{x \in \Sigma: \mathbf{U} \cdot \mathbf{n}>0\},
$$

$\Sigma_{0}=\{x \in \partial \Omega: \mathbf{U} \cdot \mathbf{n}=0\}$, où $\mathbf{n}$ est la normale sortante à $\partial \Omega=\Sigma \cup \partial S$. A son tour, le compact $\Gamma=\Sigma_{0} \cap \Sigma$ sépare la surface $\Sigma$ en trois parties disjointes $\Sigma=\Sigma_{\text {in }} \cup \Sigma_{\text {out }} \cup \Gamma$. Le problème est de trouver le champ de vitesses u et la densité de gaz $\varrho$ satisfaisant les équations suivantes ainsi que les conditions aux limites

$$
\begin{aligned}
& \Delta \mathbf{u}+\lambda \nabla \operatorname{div} \mathbf{u}=R \varrho \mathbf{u} \cdot \nabla \mathbf{u}+\frac{R}{\epsilon^{2}} \nabla p(\varrho) \text { dans } \Omega, \\
& \operatorname{div}(\varrho \mathbf{u})=0 \text { dans } \Omega, \\
& \mathbf{u}=\mathbf{U} \operatorname{sur} \Sigma, \quad \mathbf{u}=0 \text { sur } \partial S, \\
& \varrho=\varrho_{0} \text { sur } \Sigma_{\text {in }},
\end{aligned}
$$

où la pression $p=p(\varrho)$ est régulière, fonction strictement monotone de la densité, $\epsilon$ est le nombre de Mach, $R$ est le nombre de Reynolds, $\lambda$ est le taux de viscosité, et $\varrho_{0}$ est une constante positive.

Minimisation de la traînée. Une des applications principales de la théorie des écoulements visqueux compressible est le design optimal de la forme en aérodynamique. L'exemple classique en aérodynamique est le problème de minimisation de la traînée d'une aile en mouvement dans l'atmosphère avec une vitesse uniforme horizontale $\mathbf{U}_{\infty}$. On rappelle que la force aérodynamique agissant sur le corps $S$ est définie par la formule,

$$
\mathbf{J}(S)=-\int_{\partial S}\left(\nabla \mathbf{u}+(\nabla \mathbf{u})^{*}+(\lambda-1) \operatorname{div} \mathbf{u I}-\frac{R}{\epsilon^{2}} p \mathbf{I}\right) \cdot \mathbf{n} d S .
$$

Dans un cadre attaché au corps en mouvement la traînée est la composante de $\mathbf{J}$ parallèle à $\mathbf{U}_{\infty}$,

$$
J_{D}(S)=\mathbf{U}_{\infty} \cdot \mathbf{J}(S)
$$


et la portance est la composante de $\mathbf{J}$ dans la direction verticale. La minimisation de la traînée et la maximisation de la portance font partie des problèmes pratiques prioritaires en optimisation de formes aérodynamiques. Des premiers résultats sur la dépendance de $\mathbf{J}$ par rapport à la forme $S$ sont présentés dans [24] [25].

\section{Gradient}

\subsection{Gradient continu}

L'optimisation peut être entreprise sur la base d'un optimiseur ne nécessitant pas le gradient de la fonctionnelle. Néanmoins, pour des raisons d'efficacité, on peut préférer minimiser en utilisant le gradient. Les démarches ayant pour objet d'évaluer ce gradient par rapport à la forme ont fait l'objet très tôt d'avancées théoriques (Hadamard, Garabedian). Ces avancées traitaient de la sensitivité au sens classique, c'est à dire de la variation de la solution d'une Equation aux Dérivées Partielles par rapport à une donnée géométrique.

Les avancées théoriques ont continué en Contrôle Optimal. Le problème de la sensitivité de l'état a cédé la place au gradient de la fonctionnelle, exprimé en fonction de l'état adjoint. Reprenant l'exemple (2)(3) précédent, avec $W=(\rho, \rho \mathbf{u})$, mais en version Euler $(1 / R=0)$ qui s'écrit $F(W)_{x}+G(W)_{y}+H(W)_{z}=0$ dans $\Omega$, on obtient le gradient d'une fonctionnelle générique sous la forme du noyau suivant défini sur la variété $S$, cf. e.g. [5]:

$$
g_{L^{2}}(S, W, \Pi)=-\left(F(W) \frac{\partial \Pi}{\partial x}+G(W) \frac{\partial \Pi}{\partial y}+H(W) \frac{\partial \Pi}{\partial z}\right)\left(\vec{n}^{S} \cdot \vec{V}_{S}\right)+(\nabla p \Pi+p \nabla \Pi)\left(\vec{n}^{S} \cdot \vec{V}_{S}\right) .
$$

où $\Pi$ est un état adjoint défini à partir de la fonctionnelle choisie. Le vecteur $\vec{V}_{S}$ définit la direction de la perturbation de la forme $S$ et le vecteur $\vec{n}^{S}$ la normale à la forme $S$.

\subsection{Aspects numériques}

Le point de vue de la mise en oeuvre numérique a lui aussi beaucoup progressé avec un intéressant débat entre l'approche du gradient continu discrétisé et du gradient discret exact, relancé avec l'arrivée des premières expériences d'optimisation de forme en écoulements compressibles [11]. Ces "plate-formes" d'optimisation de forme ont reposé avec beaucoup d'acuité les problèmes de paramétrage et de champs de déformations, ce dernier point reposant de plus en plus sur des modèles discrets de déformation de maillage.

\section{Analyse Asymptotique et DÉRivée topologique}

Les notions de dérivées topologiques intérieures et extérieures [22], [26], [14] de fonctionnelles de formes font apparaître une famille de nouveaux problèmes mathématiques liés l'analyse asymptotique de problèmes aux limites singulièrement perturbés. Bien que plusieurs problèmes modèles traitant essentiellement du laplacien avec conditions au bord de Neumann ou de Dirichlet aient été étudiés avec succès, des classes plus générales de problèmes aux limites, y compris, par exemple, des problèmes d'élasticité bi ou tri-dimensionnels ainsi que les équations de Navier-Stokes, nécessitent une étude approfondie et le développement d'outils mathématiques de l'analyse asymptotique moderne. L'application des résultats asymptotiques à l'optimisation des formes demande généralement la connaissance de la structure asymptotique détaillée, ainsi que des estimations d'erreurs précises dans les espaces de fonctions adéquats.

Pour certains problèmes elliptiques, comme il a été démontré dans l'article [23], une variation de la topologie extérieure de la frontière est beaucoup plus avantageuse et profitable que des variations intérieures ou de la frontière. Ce genre de variations topologiques implique la formation d'un ligament fin (petit cylindre curvilinéaire) reliant deux petites régions sur la frontière en dehors du domaine original. Ce type de problèmes appelés jonctions de multi-structures, a été étudié intensivement durant les deux dernières décennies (P. Ciarlet [21], E. Sanchez-Palencia [16], [17], S.A. Nazarov, V. Maz'ya, V. Kozlov, [18], [19], [20] ...). Cependant, seules les frontières rectilignes et les cylindres ont été considérés ce qui réduit sérieusement les applications 
à l'optimisation de formes. A notre connaissance, tous les résultats asymptotiques cités auparavant sont justifiés uniquement à l'aide de l'estimation d'énergie de base qui n'est certainement pas optimale pour toute fonctionnelle de forme excepté pour l'énergie.

L'optimisation topologique n'a pas encore beaucoup touché la Mécanique des Fluides. Les outils mathématiques disponibles nous incitent à considérer des modèles visqueux ("elliptiques") avec conditions d'adhérence ("Dirichlet"), voir [2,9]. L'impact d'une nouvelle composante sera donc surtout du frottement. Pour les aérodynamiciens qui attendent plutôt de la portance, il est possible de développer une analyse topologique sur un modèle non-visqueux par exemple [13] en utilisant la formule (4). Il reste une difficulté amusante. Alors que la connexité d'une structure est contrôlée par son analyse, ce n'est absolument pas le cas en aérodynamique, qui ne fait pas la différence entre un avion en un seul ou plusieurs morceaux. Une solution pourrait être de coupler l'aérodynamique avec une analyse de la structure.

\section{A propos Des quatre COMmunications DU Mini-Symposium}

La première communication de ce minisymposium est dans la droite ligne des avancées théoriques liées à la sensitivité en paramétrage topologique et aux méthodes qui en découlement pour le contrôle optimal. Elle présente la dérivation topologique d'un système régi par une inéquation variationnelle et son adaptation à l'optimisation de forme Level Set.

Les trois communications suivantes sont plus concernées par les questions d'efficacité car elles traitent de l'optimisation de formes aérodynamiques avec des modèles qualifiés de "haute fidélité" (Euler, Navier-Stokes) qui sont particulièrement couteux dans la pratique industrielle.

La seconde communication propose un paramétrage par déformation (Free-Form-Deformation) combiné avec une représentation de la frontìere par courbes de Bézier adaptative.

La troisieme communication explore les potentialités d'une approche multiniveau reposant sur le paramétrage Bézier dans le but de réduire l'effort en nombre d'évaluation de fonctionnelles consacré à l'optimisation d'une courbe à paramétrage par discrétisation complète ("CAD-free, par opposition aux paramétrage de la CAO).

La dernière communication étudie la relation entre régularité et utilisation du gradient de forme pour des écoulements supersoniques.

\section{REFERENCES}

[1] G. Allaire, F. Jouve, A.M. Toader, A level-set method for shape optimization, C.R.A.S. Paris Sér.I, 334(2002)1125-1130.

[2] S. Amstutz, The topological asymptotic for the Navier-Stokes equations, ESAIM:COCV 11:3(2005), pp. 401-425

[3] D. Bucur, G. Buttazzo, Variational Methods in Shape ptimization Problems, Birkhäuser, 2004.

[4] D. Chenais, On the existence of a solution in a domain identication problem, J. Math. Anal. Appl. 52, pp.189-289 (1975).

[5] A. Dervieux, Y. Mesri, F. Courty, L. Hascoet, B. Koobus, M. VÀzquez, Calculs de sensibilité par différentiation pour l'Aérodynamique, ce symposium.

[6] R. Duvigneau, B. Abou El Madj, J.-A. Desideri, Towards a self adaptive parametrization for aerodynamic shape optimisation, ce symposium.

[7] Y. EgORov, On the Lagrange problem about the strongest circular column, C.R.A.S. Paris, Sér.I, 335(2002), pp.997-1002.

[8] P.R. Garabedian, Partial Differential Equations, Wiley, New-York, 1964.

[9] Ph Gulllaume, K. Sidridis, Topological sensitivity and shape optimization for the Stokes Equations, SIAM J. Control Optim., 34:1(2004), pp. 1-31.

[10] J. Hadamard, Mémoire sur le problème d'analyse relatif à l'équilibre des plaques élastiques encastrées, OEuvre de Jacques Hadamard, CNRS, Paris, 1968.

[11] A. Jameson, Aerodynamic Design via Control theory, J. Sci. Comp., 3:233-260,1988.

[12] M. Martineldi, F. Beux, Multilevel gradient based methods in aerodynamic shape design, ce symposium.

[13] M. Martinelli, F. Beux, A. Dervieux, Un gradient topologique en Aérodynamique, En préparation.

[14] S. Garreau, Ph. Guillaume, M. Masmoudi, The topological asymptotic for PDE systems: the elasticity case, SIAM Journal on Control and Optimization. 39(6)(2001) 1756-1778.

[15] F. Murat, J. Simon, Etudes de problèmes d'optimal design, Lecture Notes in Computer Science 41(1976), pp. 54-62, Springer, Berlin. 
[16] E. Sanchez-Palencia Sur certains problèmes de couplage de plaques et de barres. (French) [Some problems of the coupling of plates and rods] Équations aux dérivées partielles et applications, 725-744, Gauthier-Villars, Éd. Sci. Méd. Elsevier, Paris, 1998.

[17] D. Leguillon, E. SAnchez-Palencia, Computation of singular solutions in elliptic problems and elasticity Paris, Masson. 1987

[18] V. A. Kozlov, V. G. Maz'ya, J. Rossmann, Elliptic boundary value problems in domains with point singularities Amer. Math. Soc, Providence, 1997

[19] W. G. Mazja, S. A. Nazarov, B. A. Plamenevskil, Asymptotische Theorie elliptischer Randwertaufgaben in singulär gestörten Gebieten. 1. Berlin: Akademie-Verlag. 1991. (English transl.: Asymptotic theory of elliptic boundary value problems in singularly perturbed domains. Vol. 1, Basel: Birkhäuser Verlag, 2000)

[20] W. G. Mazja, S. A. Nazarov, B. A. PlamenevskiI, Asymptotische Theorie elliptischer Randwertaufgaben in singulär gestörten Gebieten. 2. Berlin: Akademie-Verlag. 1991. (English transl.: Asymptotic theory of elliptic boundary value problems in singularly perturbed domains. Vol. 2, Basel: Birkhäuser Verlag, 2000)

[21] P.G. Ciarlet Mathematical elasticity. Vol. II. Theory of plates. Studies in Mathematics and its Applications, 27. NorthHolland Publishing Co., Amsterdam, 1997. lxiv+497 pp. ISBN: 0-444-82570-3

[22] S. A. Nazarov, J. SokoŁowski Asymptotic analysis of shape functionals, Journal de Mathématiques pures et appliquées, 82-2(2003), 125-196.

[23] S.A. Nazarov, J. Sokolowski, The topological derivative of the Dirichlet integral under formation of a thin ligament, Siberian Math. J., 45 (2004),410-426

[24] P.I. Plotnikov, J. Sokolowski, Domain dependence of solutions to compressible Navier-Stokes equations SIAM J. Control Optim., Volume 45, Issue 4, 2006, pp. 1147-1539.

[25] P.I. Plotnikov, E.V. Ruban, J. Sokolowski, Inhomogeneous boundary value problems for compressible Navier-Stokes equations: well-posedness and sensitivity analysis, preprint 2006.

[26] J. SokoŁowski, A. Żochowski On topological derivative in shape optimization, SIAM Journal on Control and Optimization. 37, Number 4 (1999), pp. 1251-1272.

[27] V. Sverak, On optimal shape design, J. Math. Pures Appl., 72(1993), pp. 537-551.

[28] J.P. Zolesio, The material derivative (or speed) method for shape optimization, Optimization of Distributed Parameter Structures, vol. II, NATO Adv. Study Inst. Ser. E, Appl. Sci. 50 (1981) 1089-1151. 